interstellar space, the ions in the laboratory must be cooled to a state of minimum rotation and vibration. Vibrational cooling was first achieved using the CRYRING storage ring in Stockholm, Sweden, by injecting mass-selected ions into an accelerator, and allowing them to relax to their vibrational ground state. ${ }^{6}$. Achieving a low rotational temperature is more difficult: $\mathrm{H}_{3}^{+}$cannot cool rotationally by emitting radiation $^{7}$, so the ions must be prepared in a rotationally cold state before they are injected into the storage ring. AtCRYRING, this is done by sparking a discharge to ionize a jet of hydrogen gas as it expands through an opened valve into the vacuum of the storage ring and cools. Once the $\mathrm{H}_{3}^{+}$ions are in both their vibrational and rotational ground states, an electron beam travelling at a well-defined velocity is merged with the ion beam, and dissociative recombination occurs. The neutral fragments produced are counted as a function of the relative velocity of the two beams, yielding the 'spectrum' of the rate of recombination as a function of the collision energy.

Kreckel and colleagues' experiments', performed at the Test Storage Ring (TSR) in Heidelberg, Germany, follow a similar scheme, but make two improvements over the previous studies. First, they produced their electron beam with a newly developed cryogenic photocathode, allowing more precise control of the ion-electron collision energy and so higher resolution in the dissociative-recombination spectrum. Second, they used a new type of ion source, called a radiofrequency multipole ion trap. In such a trap, $\mathrm{H}_{3}^{+}$ions are stored before injection into the ring at low temperature in the presence of helium gas. The large number of collisions of the ions with the helium means that the rotational energy of the ions can be transferred to the helium, thus ensuring that the ions are rotationally cold. (In contrast, with the expanding jet source used at CRYRING, a small fraction of ions may remain rotationally warm.)

The result of the TSR experiment is in excellent agreement with the CRYRING results, and, thanks to its higher resolution, reveals the fine detail of the spectrum more clearly. It confirms that the rate of dissociative recombination is fast under cool, interstellar conditions. Improved theoretical calculations $\mathrm{s}^{3,4}$ also yield a rate that agrees well with both experiments. Some minor discrepancies remain, but the general concord implies that thelong-standing enigma of the rate of $\mathrm{H}_{3}^{+}$recombination might finally be resolved. If so, the onus is back on the astrophysicists: how can the large observed abundance of $\mathrm{H}_{3}^{+}$in diffuse clouds be explained if recombination is so fast? One likely solution would seem to be enhanced production of $\mathrm{H}_{3}^{+}$through ionization by cosmic rays ${ }^{2}$.

Kreckel and colleagues' results ${ }^{1}$ do contain an intriguing twist. At the low temperatures of their measurements (and of the interstellar medium), $\mathrm{H}_{3}^{+}$exists almost entirely in its two lowest rotational states, which have a total nuclear spin of $3 / 2$ (ortho- $\mathrm{H}_{3}^{+}$) and $1 / 2$ (para$\mathrm{H}_{3}^{+}$). But by using para- $\mathrm{H}_{2}$ in their ion source, and thus enhancing the ratio of para- to ortho$\mathrm{H}_{3}^{+}$, the authors saw a marked difference in the rate of dissociative recombination at low energies. Unfortunately, they were not able to measure the degree of the para- $\mathrm{H}_{3}^{+}$enhancement, and because of the nature of their ion source, it is probably not very large. Future experiments with pure para- $\mathrm{H}_{3}^{+}$would be highly desirable to elucidate the difference in the rate of recombination between the two states. That would indeed represent the first dissociative recombination measurement of a single quantum state.
Benjamin J. McCall is in the Departments of Chemistry and Astronomy, University of Illinois at Urbana-Champaign, 600 South Mathews Avenue, Urbana, Illinois 61801, USA. e-mail:bjmccall@uiuc.edu

1. Kreckel, H.et al. Phys. Rex Lett. 95, 263201 (2005).

2. McCall, B. L et al Nature 422,500-502 (2003).

3 Kokoouline, $V_{7}$ Greene, C. H. \& Esry, B. D. Nature 412, 891-894 (2001).

4. Koloouline, V.\& Greene, C. H. Phys. Rex A68, 012703 (2003)

5. Larsson, M. Phl. Trans. R. Soc. Lond A 358,2433-2444 (2000)

6. Larsson M. etal. Phys. Rex Lett. 70,430-433 (1993).

7. Kreckel, H.et al. New J.Phys. 6, 151(2004).

8. MoCall, B.l etal Phys Rev.A70,052716 (2004).

\title{
NEUROBIOLOGY
}

\section{How fast can you go?}

\author{
Laura N. Borodinsky
}

\section{Rhythmic activities such as walking need tight coordination. In mice, pace is tweaked by a specific set of spinal-cord neurons that, surprisingly, make the animals walk faster by inhibiting the underlying circuit.}

Watch your step - walking may seem simple, but is actually quite a complex task. As with other rhythmic motor behaviours (breathing or swallowing, say), locomotion relies on a finely tuned neuronal network that is headquartered in the spinal cord ${ }^{1,2}$. The ensemble of spinal neurons that generates a coordinated rhythmic activity is known as a central pattern generator. The rhythm and periodicity of this network determines movement features such as the alternation between left and right, or the speed of walking, jumping or swimming ${ }^{3}$. Understanding how this circuit operates and the specific roles of the different neurons that participate in it is difficult, but Gosgnach and colleagues ${ }^{4}$ have taken up the challenge. On page 215 of this issue, they report that the activity of a group of spinal-cord neurons controls the speed of locomotor behaviour in the mouse.

Neuronal circuits are formed by a network of interconnected excitatory and inhibitory neurons. In a very simplistic model, the former group switches the circuit on and the latter turns it off. Gosgnach et al. studied the role of a subclass of inhibitory spinal neurons known as V1 neurons, which are thought to be part of the central pattern generator. The researchers used detailed information about the gene-regulatory factors that underlie the development and specialization of these neurons, to generate mutant mice in which V1 neurons were either eliminated or silenced acutely during the experiment, and examined the changes in locomotor activity.

Counterintuitively, they found that removing the inhibition caused by V1 neurons slows the speed of the locomotor rhythm. Mutant mice lacking V1 neurons are unable to walk fast, but they can maintain normal motor behaviour at a slower pace. The authors demonstrate that this is because motor neurons connecting the spinal cord to the muscle are not sufficiently inhibited, because connections from the V1 neurons are missing in the mutant animals. The activity of motor neurons needs to be interleaved with precise periods of silence to generate a faster pace. These results underscore the value of inhibition in the nervous system: the delicate balance and fine-tuning of neuronal activity set by inhibitory connections is not only important in quieting down the system, but can also change core features of nervous-system function.

Even though Gosgnach and colleagues' mice slow down, other parameters of their locomotor activity remain intact, such as the alternation of left and right limbs necessary for coordinated stepping. As the authors showed previously ${ }^{5}$, a different class of spinal neurons (V0) is responsible for left-right coordination. In mutant mice lacking V0 neurons, the left and right motor neurons fire at the same time, rather than alternating. These are significant insights into how the work is distributed among the vast collection of spinal neurons that make up the central pattern generator.

How are the circuits of the central pattern generator established? When exploring the formation of circuits, scientists have focused on two principal alternative theories. One of these proposes that a genetically driven programme predetermines the identity of the neurons that participate in a given circuit and dictates how and between which of them connections are made ${ }^{6}$. The second proposes that 
early in development, neurons exhibit spontaneous electrical activity that affects the process of neuronal specialization and the establishment of appropriate connections ${ }^{7.8}$. Changes in activity during development can indeed lead to resetting of the intrinsic excitability of neurons as well as reconfiguration of connections'. In particular, formation of motor central pattern generators requires early spontaneous electrical activity, because when this activity is disrupted, motor neuron fibres fail to follow their normal trajectories ${ }^{10}$.

However, Gosgnach et al. ${ }^{4}$ find that the activity of V1 neurons does not seem to be involved in the formation of the central pattern generator. Their results show that loss of V1 neurons early in development is not compensated for by any reconfiguration of the circuit - the mutant mice that chronically lost V1 neurons at early stages exhibit the same slow locomotor rhythm as that observed when neurons are acutely silenced after the circuit is formed.

Nevertheless, studies of rewiring during development are complex; they require a rather dynamic approach because exclusive examination of the final end point may not reveal intermediate remodelling processes that are crucial to the resulting network. In this regard, it would be interesting to test what happens to the circuit and locomotor activity if neurons are silenced at different times during development. Formation of circuits is likely to depend on both genetically driven and activity-shaped processes. We need to keep our minds open if we are to understand the interplay of these driving forces.

Developmental biologists and physiologists have tended to approach the problem of circuit formation and function from very different angles, without much dialogue between them. But genetic tools are now available to reconcile the results from these two disciplines and establish a fruitful interaction. Information about signatures of gene-regulatory factors that determine the fates of neuronal populations can now serve as tools with which to elucidate the functions of a given set of neurons. The work presented by Gosgnach et al. sets up a firm bridge across the river that has so far divided developmental biology and physiology.

Laura N. Borodinsky is in the Neurobiology

Section, Division of Biological Sciences, University of California, San Diego, 9500 Gilman Drive, La Jolla, California 92093-0357, USA. e-mail: Iborodin@biomail.ucsd.edu

1. Sherrington, C. J.Physiol. (Lond) 47,196-214 (1913).

2. Hamburger, $V_{\gamma}$ Wenger, E.\& Oppenheim, R.J. Exp Zool. $162,133-160$ (1966).

3 Brown T. Proc. R. Soc. Land B 84, 308-319 (1911).

4. Gosgnach, S.etal. Nature 440,215-219 (2006).

5 Lanuza, G.M. Gosgnach, S, Plerani, A, lessell, T.M.\& Goulding M.Neuran 42, 375-386 (2004).

6. Tanabe, Y \& lessell, T. M. Science 274,1115-1123(1996).

7. Gu X.\& Spitzer, N.C. Nature375, 784-787(1995)

8. Catalano, S.M. \& Shatz, C.J.Science 281, 559-562 (1998),

9. Turrigiano, G.G. \& Nelson, S.B. Nature Rev. Neurasci.5, 97-107(2004).

10. Hanson, M. G \& Landmesser, L. T. Neuran 43, 687-701 (2004).

\title{
BIOENGINEERING
}

\section{Diagnosis on disc}

\author{
Frances S. Ligler and Jeffrey S. Erickson
}

\section{Highly complex immunoassays that identify and quantify many different antigens simultaneously need high-resolution imaging capability. A simple, low-cost technique could be music to our ears.}

The capabilities of immunoassays - tests that use the binding of 'capture' antibodies to antigens in order to identify the latter - have been advancing in leaps and bounds over the past three decades. Methods of enzyme amplification have increased assay sensitivity. Diode lasers have reduced the size and cost of instrumentation. Microfluidics has enabled both the analysis of very small sample volumes and the parallel processing of multiple samples. And microarrays of capture antibodies, or other recognition molecules, attached to surfaces have made possible the simultaneous testing of a sample for large numbers of target molecules.

This last point harbours a problem. As microarray elements become smaller and smaller for larger and larger numbers of simultaneous tests, the necessary imaging resolution cannot readily be achieved with the standard, off-the-shelf CMOS or CCD technologies. Confocal scanners using microscope lenses can provide the required resolution, but their cost, size and the geometrical alignment of their optics pose other practical problems. Writing in Angewandte Chemie International Edition, Sebastian A. Lange, Günter Roth and colleagues find a way out of this seeming impasse ${ }^{1}$. They demonstrate an immunoassay readout of high efficiency and sensitivity using, in a slightly modified form, a pick-up head of a compact-disc player (Fig. 1).

The authors' system is both elegant and simple. They used a so-called sandwich immunoassay, in which the antigen to be measured is bound between two different antibodies. First, a capture antibody is stamped in a 25-micrometre square pattern onto a solid substrate; the desired antigen binds to this antibody. Second, a detector antibody binds to this antigen to make it visible to the $\mathrm{CD}$ pickup head. Here, the authors cleverly employed an antibody carrying a gold nanoparticle. This gold nanoparticle catalyses the deposition of silver grains onto the substrate to which the capture antibody, antigen and detector antibody are all now attached. The reflections from the silver grains could be read by the $C D$ pick-up head with a resolution of around 50 nanometres - considerably smaller than the diameter of most of the silver nanoparticles, which is of the order of several hundred nanometres. Using their sandwich system, the authors demonstrated that they could detect antigen in serum at concentrations from 1 microgram per millilitre down to 100 picograms per millilitre.

The authors also performed a carefully controlled study in which they spaced antigens, each attached to a capture molecule, well apart from each other on the substrate ${ }^{1}$. They suggest that, by correlating the density of the silver precipitate with the coverage of the antigen, it might be possible to detect single molecules with their system. But measuring a single molecule carefully tied to a uniform surface is a far cry from pulling a single molecule out of a solution, capturing it at a surface and measuring it against a background signal generated from any of several variable sources. The potential for using the $C D$ pick-up head with silver staining for single-molecule detection is therefore less convincing than the suggestion that the technology could be widely useful for low-cost, high-sensitivity readout
Figure 1|Molecularmusic. A conventional compact-disc pick-up reader works by focusing laser light onto the surface of the CD. As the CD rotates above the reader, information encoded as pits along a spiral track on its metal-coated surface can be read by means of light reflected back through a lens onto a photodiode. Lange and colleagues' immunoassay system ${ }^{1}$ works in exactly the same way, but with a substrate covered with a regularly spaced array of capture antibodies taking the place of the CD. When an antigen binds to an antibody, its presence is signalled by a second, gold-containing detector antibody that catalyses the deposition of reflective silver particles onto the substrate.

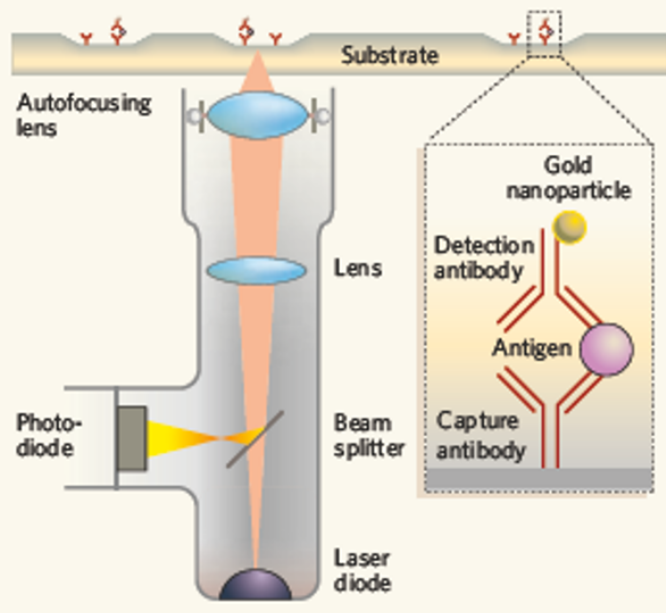

